\title{
A Hospitalização Domiciliária na Pandemia COVID-19
}

\section{Home Hospitalization in the COVID-19 Pandemic}

Pedro Correia Azevedo ${ }^{1}$

Autor Correspondente/Corresponding Author:

Pedro Azevedo [pedro.azevedo@jmellosaude.pt] Rua Manuel Tito Morais, $n^{\circ}$ 2, 2825-146 Caparica, Portugal

ORCID iD: 0000-0002-2630-2221

\section{RESUMO}

A hospitalização domiciliária tem vindo a implementar-se no sistema de saúde português como um modelo de assistência de nível hospitalar no domicílio. É uma alternativa ao internamento convencional, para doentes agudos ou crónicos agudizados, sendo a admissão nesta tipologia de internamento voluntária. Devem estar garantidos os critérios de admissão, que passam essencialmente pela estabilidade clínica com comorbilidades controláveis no domicílio, presença de cuidador e domicílio com boas condições de higiene e segurança, e domicílio na área de influência da unidade hospitalar de referência. As vantagens da hospitalização domiciliária parecem ser evidentes, nomeadamente no que toca à significativa redução das infeções nosocomiais, quedas e episódios de síndroma confusional aguda. No atual contexto da pandemia COVID-19, a hospitalização domiciliária pode ser parte da solução de resposta do sistema de saúde, não só pelo aumento da capacidade de internamento domiciliário de doentes sem infeção por SARS-CoV-2 mas também, cumprindo todas as recomendações de segurança, ser uma possibilidade de internamento para doentes com infeção por SARS-CoV-2 após, pelo menos, 7 dias de internamento hospitalar convencional com estabilidade clínica, possibilidade de isolamento e cuidador não portador de condição de saúde vulnerável. A equipa de saúde da hospitalização domiciliária assume aqui um papel-chave na educação e promoção da saúde, proporcionando aprendizagens no que toca às medidas de isolamento, higiene das mãos e etiqueta respiratória.

Cumpridos os critérios de admissão, a hospitalização domiciliária é uma tipologia de internamento que permite atingir a eficiência sem prejuízo da segurança do doente.

PALAVRAS-CHAVE: COVID-19; Hospitalização; Pandemia; SARS-CoV-2; Serviços Hospitalares de Assistência Domiciliar; Telemedicina

1. Diretor Clínico dos Cuidados Domiciliários CUF, Lisboa, Portugal; Coordenador da Medicina Interna e do Atendimento Permanente de Adultos da Clínica CUF-AImada, Almada, Portugal; Assistente Hospitalar de Medicina Interna - Serviço de Medicina Interna e Unidade de Hospitalização Domiciliária do Hospital Garcia de Orta, Almada, Portugal.

Recebido/Received: 07/06/2020 - Aceite/Accepted: 14/06/2020 - Publicado/Published: 30/06/2020

๑ Autor (es) (ou seu (s) empregador (es)) 2020. Reutilização permitida de acordo com CC BY-NC. Nenhuma reutilização comercial. ${ }^{\bullet}$ Author(s) (or their employer(s)) 2020. Re-use permitted under CC BY-NC. No commercial re-use. 


\section{ABSTRACT}

Home hospitalization has been implemented in the Portuguese health system as a model of hospital-level assistance at home. It is an alternative to conventional hospitalization for acute or acute-on-chronic patients. The admission to this type of hospital care is voluntary. Admission criteria must be guaranteed: clinical stability with controllable comorbidities at home, presence of caregiver and home with good hygiene and safety conditions, home in the area of influence of the referral hospital. The advantages of home hospitalization seem to be evident, namely with regard to the significant reduction in nosocomial infections, falls and episodes of acute confusional syndrome.

In the current context of the COVID-19 pandemic, home hospitalization can be part of the health system's response solution, not only by increasing the home care capacity of patients without SARS-CoV-2 infection, but also, complying with all the recommendations of safety, be a possibility of hospitalization for patients with SARS-CoV-2 infection after at least 7 days of conventional hospitalization with clinical stability, possibility of isolation and caregiver without vulnerable health condition. The health team of home hospitalization assumes a key role in health education, teaching in terms of isolation measures, hand hygiene and respiratory etiquette.

Once the admission criteria are met, home hospitalization is a type of hospitalization that allows efficiency to be achieved without prejudice to patient safety.

KEYWORDS: COVID-19; Home Care Services, Hospital-Based; Hospitalization; Pandemics; SARS-CoV-2; Telemedicine

\section{DEFINIÇÃO E CONTEXTO DA HOSPITALIZAÇÃO DOMICILIÁRIA}

A hospitalização domiciliária (HD) é uma alternativa ao internamento convencional que se caracteriza pela prestação de cuidados de saúde de nível hospitalar no domicílio. ${ }^{1,2}$ Este internamento tem complexidade, intensidade e duração equivalentes ao internamento convencional. Os doentes elegíveis para a HD são aqueles que cumprem os requisitos nas seguintes categorias ${ }^{2-6}$ :

- Clínico - situação clínica transitória (doença aguda ou crónica agudizada) com diagnóstico conhecido e estabilidade clínica que permite a permanência no domicílio, onde é realizada a visita diária da equipa de saúde (médico, enfermeiro e, eventualmente, outros profissionais);

- Social - o domicílio tem de ter boas condições de higiene e segurança; tem de existir possibilidade de contacto telefónico; é necessária a presença de um cuidador, que pode assumir um papel muito importante na vigilância do doente;

- Geográfico - o domicílio tem de estar na área de influência da unidade hospitalar de referência.

A aceitação da admissão neste modelo de internamento é voluntária, com a assinatura do consentimento informado por parte do doente e/ou representante legal. ${ }^{3}$

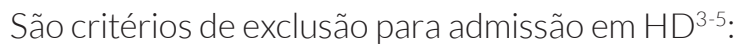

- Uso de substâncias ilícitas (nomeadamente endovenosas);
- Patologia psiquiátrica aguda;

- Pessoa indigente ou sem-abrigo;

- Incapacidade física e/ou mental dos doentes ou cuidadores que condicione a compreensão dos cuidados necessários e tratamentos prescritos.

A equipa de saúde visita diariamente os doentes, numa experiência de abordagem conjunta entre médico e enfermeiro, não tão frequente no internamento convencional. É feita a observação diária dos doentes e os ajustes farmacológicos necessários. A equipa está disponível 24 horas para responder em caso de dúvida ou agudização clínica, sendo o contacto realizado por telefone, que substitui a campainha do quarto do hospital.,37 No contexto da Medicina moderna, o uso de dispositivos de telemonitorização pode ser encarado como um parceiro na vigilância ativa dos doentes internados no domicílio e já existem várias soluções disponíveis que, inclusivamente, estão associadas a uma redução dos custos com a hospitalização. ${ }^{8}$

Este modelo de assistência hospitalar apresenta-se como uma oportunidade única de entender a pessoa doente no seu meio habitual, permitindo o ajuste das abordagens farmacológicas e não-farmacológicas à realidade individual. Permite, assim, a criação de oportunidades únicas de educação e promoção da saúde no contexto habitual da pessoa e assiste-se, muitas vezes, a uma adaptação no controlo das comorbilidades. Em acréscimo, parece haver uma redução clara das infeções nosocomiais, úlceras de pressão e quedas secundárias a 
longos períodos de imobilização, síndroma confusional aguda e declínio do estado funcional e de desnutrição situações particularmente preocupantes nos idosos. ${ }^{9}$

A Medicina Interna, como especialidade médica holística e hospitalar, assume um papel-chave no seguimento destes doentes. A experiência demonstra que os médicos internistas, em conjunto com a enfermagem, são capazes de criar um envolvimento exclusivo particular com os doentes e os cuidadores em todo o processo de internamento, contribuindo para a educação e literacia da comunidade e para uma maior adesão aos cuidados de saúde e à terapêutica. ${ }^{10}$

Das várias experiências conhecidas de HD, em países diferentes e com grupos diferentes de doentes, pode-se genericamente considerar a HD uma alternativa válida por permitir:

- Controlar a doença de forma personalizada no ambiente do doente ${ }^{11}$;

- Reduzir custos pelo melhor controlo da doença e pelo menor período efetivo de acompanhamento médico e de enfermagem necessários ${ }^{12}$;

- Reforçar a humanização da Medicina com enfoque na comunicação médico-doente";

- Melhorar a articulação com o médico assistente9;

- Melhorar a qualidade do sono dos doentes e estar associado a menor tempo de imobilização9;

- Melhorar a satisfação dos doentes e cuidadores em relação ao sistema de saúde, pela melhor perceção da doença pela família e pelo maior conforto do doente $1,6,12$;

- Reduzir o número de infeções nosocomiais e complicações $^{4,6,9}$;

- Reduzir o tempo de internamento.6,11

A responsabilização dos doentes e dos cuidadores, integrando-os no processo de vigilância e internamento, poderia ser encarado como uma desvantagem, contudo, a experiência tem demonstrado que esta aplicação dos princípios básicos da ética médica tem sido uma mais-valia deste modelo. 2,5,13

Originalmente, este modelo de internamento surgiu na década de 40 do século XX, nos Estados Unidos da América, como uma possibilidade de resposta à falta de camas nos hospitais. Atualmente, já com larga implementação na Europa, os modelos não são uniformes naquilo que consideram como HD, englobando cuidados de enfermagem no domicílio, referenciação direta a partir dos serviços de urgência e/ou comunidade sem admissão hospitalar prévia, alta hospitalar precoce com continuidade do seguimento no domicílio, entre outros. 2,6,10,12,14

Em Portugal, a HD surgiu em 2015 no Hospital Garcia de Orta, decalcando o modelo espanhol em vigor desde 1981 (iniciado no Hospital Universitário Gregório Marañon), sendo uma extensão do hospital no domicílio e aproximando o hospital da comunidade - fala-se assim de hospital at home, um modelo particular de homecare. ${ }^{15}$ Esta prestação de cuidados assenta em 5 princípios fundamentais ${ }^{7}$ :

1. Voluntariedade na aceitação do modelo por parte dos doentes e cuidadores;

2. Igualdade de direitos e deveres dos doentes;

3. Equivalência da qualidade na prestação dos cuidados;

4. Rigor na admissão dos doentes e seguimento clínico;

5. Humanização de serviços e valorização do papel da família.

No domicílio realizam-se vários exames complementares de diagnóstico, nomeadamente análises de sangue e urina, eletrocardiograma, paracentese, entre outros. No caso de haver necessidade de realizar um exame não passível de ser realizado no domicílio, ou em caso de agudização com necessidade de um nível superior de vigilância, após validação pela equipa de saúde, o transporte à unidade hospitalar de referência deve estar garantido. 9,10

Nas unidades de HD em Portugal tem-se assistido a formação pré- e pós-graduada de médicos, enfermeiros, técnicos superiores de serviço social e farmacêuticos. ${ }^{10}$ Contribui-se, assim, para a implementação e expansão do conceito e para a evolução da ciência.

O modelo português, iniciado no Serviço Nacional de Saúde, está agora em expansão por todo o Sistema de Saúde e deve promover-se a criação de condições para que esta reposta de internamento possa ser cada vez mais difundida. As patologias mais frequentemente internadas em HD são semelhantes às que se conhecem do internamento convencional ao cargo da especialidade de Medicina Interna?:

- Infeções respiratórias;

- Infeções urinárias;

- Infeções da pele e tecidos moles;

- Insuficiência cardíaca;

- Infeção de feridas operatórias e próteses.

Tendo em conta estes diagnósticos, infere-se a necessidade de uma política antimicrobiana rigorosa e o de- 
senvolvimento de protocolos de Tratamento Antimicrobiano Domiciliário Endovenoso (TADE). O farmacêutico assume aqui um papel ativo na equipa da HD. 10,16,17

Desta maneira, a HD apresenta-se como um modelo de prestação de cuidados hospitalares, no domicílio, centrado no doente, sem as complicações inerentes à hospitalização convencional.

\section{A HOSPITALIZAÇÃO DOMICILIÁRIA COMO PARTE DA RESPOSTA À PANDEMIA SARS-CoV-2}

A 31 de dezembro de 2019 foi declarada a existência de vários casos de pneumonia de etiologia desconhecia em Wuhan, uma cidade da província de Hubei, na China. Uma semana mais tarde surgiu a confirmação de que se tratava de uma doença de etiologia viral causada pelo novo coronavírus (SARS-CoV-2) e tem-se vindo a assistir à disseminação desta doença - a 11 de março de 2020 foi declarada a pandemia COVID-19. A doença causada por este vírus pode ter várias formas de manifestação, desde quadros clínicos gripais ligeiros até quadros de pneumonia grave com necessidade de abordagem pela Medicina Intensiva. A maioria dos casos notificados de COVID-19 são quadros leves, requerendo apenas tratamento sintomático e vigilância de sintomas. ${ }^{18-21}$

Em HD, as infeções respiratórias representam cerca de 30\% dos internamentos, tendo normalmente associada insuficiência respiratória parcial com possível indicação para oxigenoterapia suplementar e, eventualmente, terapêutica antibiótica e cinesiterapia respiratória. ${ }^{10,22}$

Considerando a atual pandemia SARS-CoV-2, a apresentação clínica mais frequente desses doentes e a forma de propagação, a HD pode ter um papel importante na organização do sistema de saúde na resposta à pandemia, de resto como afirmado pelo Documento de Consenso de Peritos sobre o Posicionamento da Hospitalização Domiciliária como Estratégia Integrada no Plano Nacional de Preparação e Resposta para a Doença por Coronavírus (COVID-19) (Núcleo de Estudos de Hospitalização Domiciliária da Sociedade Portuguesa de Medicina Interna)23,24.

1. Num primeiro momento, a HD pode ser uma alternativa válida para doentes sem infeção por SARS-CoV-2 ao permitir que os hospitais adquiram mais capacidade de resposta no internamento convencional para situações que necessitem de maior vigilância. A admissão de doentes na HD poderá ser realizada diretamente em casa por referenciação do médico assistente. Os contactos do médico assistente com os doentes através de alternativas aos modelos clássicos, como seja a teleconsulta, trazem aqui uma vantagem acrescida. Estes modelos de resposta, a revelarem-se vantajosos, deverão ser encarados como soluções a perdurar no Sistema de Saúde;

2. Num segundo momento, a HD pode ser parte da resposta à infeção por SARS-CoV-2 com a possibilidade do internamento de doentes com COVID-19 em regime domiciliário, de acordo com a norma da Direção- Geral da Saúde n. 004/2020: após, pelo menos, 7 dias de internamento convencional; com estabilidade clínica e evolução favorável; se necessidade de oxigénio suplementar apenas com débito de igual ou inferior a $2 \mathrm{l} / \mathrm{min}$; com possibilidade de isolamento em casa; com cuidador não portador de condição de saúde vulnerável. Neste contexto, a telemonitorização é uma ferramenta muito importante, devendo ser entregues aos doentes dispositivos que permitam a monitorização de sinais vitais e a possibilidade de alertas urgentes;

3. Por fim, num terceiro momento, caso a capacidade do sistema de saúde esteja esgotada, a HD poderá ser uma alternativa de internamento primário para casos confirmados ou prováveis de infeção por SARS-CoV-2.

Neste contexto pandémico, o trabalho do Técnico Superior de Serviço Social reveste-se de particular importância, na avaliação das condições de isolamento domiciliário e na procura de respostas sociais na comunidade, planeando precocemente a alta do doente. Em todos os momentos devem os médicos e enfermeiros realizar o ensino para reconhecimento de sinais de agravamento respiratório. Nunca poderão ser esquecidas as medidas de higiene e segurança de doentes, cuidadores e profissionais de saúde, com o fornecimento e uso adequado de equipamentos de proteção individual. Devem ser sempre asseguradas medidas de isolamento no domicílio, com a possibilidade de lavagem das mãos, ensino e promoção da etiqueta respiratória, limpeza e desinfeção do espaço físico e contenção da movimentação em casa. ${ }^{10,25}$ A criação de protocolos de atuação específicos pelas instituições é recomendável.

Antes, durante e após a pandemia COVID-19, a Hospitalização Domiciliária é parte da solução global do sistema de saúde, expandindo o hospital para a comunidade e constituindo um modelo de internamento que permite atingir a eficiência sem prejuízo da segurança do doente. A Medicina Interna é a especialidade médica que permite aplicar o dinamismo que este modelo de internamento exige. 
AGRADECIMENTOS/

ACKNOWLEDGEMENTS

- Dra. Francisca Delerue - Diretora do Serviço de Medicina Interna e da Unidade de Hospitalização Domiciliária do Hospital Garcia de Orta.

- UHD do HGO - Unidade de Hospitalização Domiciliária do Hospital Garcia de Orta.

- NEHospDom da SPMI - Núcleo de Estudo de Hospitalização Domiciliária da Sociedade Portuguesa de Medicina Interna.

\section{RESPONSABILIDADES ÉTICAS}

CONFLITOS DE INTERESSE: Os autores declaram a inexistência de conflitos de interesse na realização do presente trabalho.

FONTES DE FINANCIAMENTO: Não existiram fontes externas de financiamento para a realização deste artigo.

PROVENIÊNCIA E REVISÃO POR PARES: Não comissionado; revisão externa por pares.

\section{ETHICAL DISCLOSURES}

CONFLICTS OF INTEREST: The authors have no conflicts of interest to declare.

FINANCING SUPPORT: This work has not received any contribution, grant or scholarship.

PROVENANCE AND PEER REVIEW: Not commissioned; externally peer reviewed.

\section{REFERÊNCIAS}

1. Maaravi Y, Cohen A, Hammerman-Rozenberg R, Stessman J. Home hospitalization. J Am Med Dir Assoc. 2002;3:114-8.

2. Voudris KV, Silver MA. Home Hospitalization for Acute Decompensated Heart Failure: Opportunities and Strategies for Improved Health Outcomes. Healthcare. 2018;6:31. doi: 10.3390/healthcare6020031.

3. Direção-Geral da Saúde. Hospitalização Domiciliária em idade adulta. Norma n. 020/2018 20/12/2018. 2018:1-22. [consultado em junho de 2020]. Disponível em: https://www.dgs.pt/ directrizes-da-dgs/normas-e-circulares-normativas/norma-n-0202018-de-20122018-pdf.aspx.

4. Salazar A, Estrada C, Porta R, Lolo M, Tomas S, Alvarez M. Home hospitalization unit: An alternative to standard inpatient hospitalization from the emergency department. Eur J Emerg Med. 2009;16:121-3. doi:10.1097/MEJ.0b013e32831cbae2.

5. Tibaldi V, Isaia G, Scarafiotti C, Gariglio F, Zanocchi M, Bo $M$, et al. Hospital at home for elderly patients with acute decompensation of chronic heart failure. Arch Intern Med. 2009;169:1569-75. doi:10.1001/archinternmed.2009.267.

6. Leff B, Burton L, Mader SL, Naughton B, Burl J, Inouye SK, et al. Hospital at home: feasibility and outcomes of a program to pro- vide hospital-level care at home for acutely ill older patients. Ann Intern Med. 2005;143:798-808. doi: 10.7326/00034819-143-11-200512060-00008.

7. Unidade de Hospitalização Domiciliária do Hospital Garcia de Orta. Programa Funcional 2018 da Unidade de Hospitalização Domiciliária do Hospital Garcia de Orta [consultado em junho de 2020]. Disponível em: http://www.hgo.pt/Portals/O/Documentos/UHD_Plano_Funcional_2018.pdf.

8. Mirón Rubio M, Ceballos Fernández R, Parras Pastor I, PaIomo Iloro A, Fernández Félix BM, Medina Miralles J, et al. Telemonitoring and home hospitalization in patients with chronic obstructive pulmonary disease: study TELEPOC. Expert Rev Respir Med. 2018;12:335-43. doi: 10.1080/17476348.2018.1442214.

9. Cunha V, Escarigo MC, Correia J, Nortadas R, Azevedo PC, Beirão P, et al. Hospitalização Domiciliária: Balanço de um Ano da Primeira Unidade Portuguesa. Med Interna. 2017;24:2905. doi: 10.24950/rspmi/O112/17/2017.

10. Delerue F, Correia J. Hospitalização Domiciliária mais um Desafio para a Medicina Interna. Med Interna. 2018:25:15-7. doi: 10.24950/rspmi/Op/1/2018

11. Alves M, Bigotte Vieira M, Costa J, Vaz Carneiro A. Análise da Revisão Cochrane: Alta Hospitalar Precoce para Hospitalização Domiciliária. Acta Med Port. 2017:30:835-9. Portuguese. doi: 10.20344/amp.9791.

12. Varney J, Weiland TJ, Jelinek G. Efficacy of hospital in the home services providing care for patients admitted from emergency departments: an integrative review. Int J Evid Based Healthc. 2014;12:128-41. doi: 10.1097/XEB.0000000000000011.

13. Leff B, Burton L, Mader SL, Naughton B, Burl J, Koehn D, et al. Comparison of stress experienced by family members of patients treated in hospital at home with that of those receiving traditional acute hospital care. J Am Geriatr Soc. 2008;56:11723. doi: 10.1111/j.1532-5415.2007.01459.x.

14. Maru S, Byrnes J, Carrington MJ, Chan YK, Thompson DR, Stewart S, Scuffham PA; WHICH? Trial Investigators. Cost-effectiveness of home versus clinic-based management of chronic heart failure: Extended follow-up of a pragmatic, multicentre randomized trial cohort - The WHICH? study (Which Heart Failure Intervention Is Most Cost-Effective \& Consumer Friendly in Reducing Hospital Care). Int J Cardiol. 2015;201:368-75. doi: 10.1016/j.ijcard.2015.08.066.

15. Cotta R, Suárez-Varela M, González A., Cotta J, Real E, Ricós J. La hospitalización domiciliaria: antecedentes, situación actual y perspectivas. Rev Panam Salud Publica/Pan Am J Public Health. 2001;10:45-55.

16. Baharoon S, Almodaimeg H, Al Watban H, Al Jahdali H, Alenazi T, Al Sayyari A, Al Dawood A, Al-Sultan M, Al Safi E. Home intravenous antibiotics in a tertiary care hospital in Saudi Arabia. Ann Saudi Med. 2011;31:457-61. doi: 10.4103/02564947.84621.

17. Estrada Cuxart O, Cuxart Mèlich A, Bonet Papell G, Riera Riezu C. Tratamiento antibiótico por vía intravenosa domiciliario y hospitalización a domicilio. Med Clin. 2007:128:798. doi: 10.1157/13106334.

18. Wanden-Berghe C, Sanz-Valero J. La Hospitalización a Domicilio en la enfermedad del COVID-19. Hosp Domic. 2020;4:557. doi: 10.22585/hospdomic.v4i2.105.

19. Martínez AM, de los Cobos JR, Castellano MR, Rubio MM, Ozamiz AB, Cuxart OE, et al. Derivación a hospitalización a domicilio de pacientes con infección por COVID-19. Hosp Domic. 2020;4:59-67. doi: 10.22585/hospdomic.v4i2.104.

20. Zhou F, Yu T, Du R, Fan G, Liu Y, Liu Z, et al. Clinical course and risk factors for mortality of adult inpatients with $\mathrm{CO}$ - 
VID-19 in Wuhan, China: a retrospective cohort study. Lancet. 2020;395:1054-62. doi: 10.1016/S0140-6736(20)30566-3.

21. Direção-Geral da Saúde. Doença pelo novo Coronavírus (COVID-199 - Nova definição de caso. Norma n. 02A/2020 25/01/2020, atualizada em 9/03/2020, atualizada em 04/03/2020. 2020:1-2. [consultado em junho de 2020]. Disponível em: https://www.dgs.pt/saude-ocupacional/documentos-so/orientacao_02_2020-pdf.aspx.

22. Nortadas R, Azevedo PC, Cunha V, Delerue F. A Hospitalização Domiciliária e a COVID-19. Med Interna 2020;COVID-19:1-3. doi: 10.24950/rspmi/ COVID19/HGO/S/2020.

23. Nortadas R, Azevedo PC, Pereira C, Cunha V, Escarigo C, Mendes D, et al. Documento de Consenso de Peritos sobre o Posicionamento da Hospitalização Domiciliária como Estratégia Integrada no Plano Nacional de Preparação e Resposta para a Doença por Coronavírus (COVID-19) - Núcleo de Estudos de Hospitalização Domiciliária da Sociedade Portuguesa de Medicina Interna. 2020:1-4. [consultado em junho de 2020]. Disponível em: https://www.spmi.pt/wp-content/ uploads/2019/06/Documento-de-Consenso-Hospitalizac\%CC\%A7a\%CC\%83o-Domicilia\%CC\%81ria-Plano-Contige\%CC\%82ncia-COVID19-.pdf.

24. Direção-Geral da Saúde. COVID-19: FASE DE MITIGAÇÃO. Abordagem do Doente com Suspeita ou Infeção por SARS-CoV-2. Norma n. 004/2020 23/03/2020, atualizada em 25/04/2020. 2020:1-2. [consultado em junho de 2020]. Disponível em: https://www.dgs.pt/saude-ocupacional/documentos-so/orientacao_02_2020-pdf.aspx.

25. World Health Organization. Home care for patients with suspected novel coronavirus (COVID-19) infection presenting with mild symptoms, and management of their contacts: interim guidance, 04 February 2020. World Health Organization. 2020:1-4. [consultado em junho de 2020]. Disponível em: https://apps.who.int/iris/handle/10665/331133. 